\title{
COLOMBIA 2000-2013: RELACIONES ENTRE CRECIMIENTO ECONÓMICO Y COMERCIO INTERNACIONAL
}

\author{
Por: Giovanni E. Reyes - Isabella Loaiza Saa ${ }^{1}$
}

\section{RESUMEN}

Este estudio realiza un análisis de las principales variables económicas de Colombia, basándose en el comportamiento de las mismas en la historia inmediata, específicamente el período bajo estudio es de 2000 a 2013. Las variables con mayor análisis son el crecimiento económico y el comercio exterior. No obstante, a fin de tener una mejor perspectiva se incorporan la tasa anual de inflación y el desempleo. En particular luego de la implementación de las políticas económicas basadas en el "Consenso de Washington" en Colombia, como en muchos países latinoamericanos, se han privilegiado las exportaciones como motores de crecimiento. El principal argumento de este documento es que -más que la cantidad de productos exportados- lo que mayormente ha influido ha sido el precio o factura de los productos vendidos en el exterior.

Palabras clave: negociaciones comerciales internacionales, economía colombiana, condiciones económicas y sociales de Colombia.

Clasificación JEL: E01, E10, F14.

1. Giovanni E. Reyes, Ph.D. University of Pittsburgh/Harvard; Profesor Titular, Escuela de Administración, Universidad del Rosario, Colombia.

Isabella Loaiza Saa, investigadora integrante del Harvard's Rethinking the MBA in Latin America, y del Centro Libertad y Emprendimiento.

Fecha de recepción: 22 de febrero de 2014 - Fecha de aprobación definitiva: 21 de abril de 2014 


\title{
COLOMBIA 2000-2013: RELATIONSHIP BETWEEN ECONOMIC GROWTH AND INTERNATIONAL TRADE
}

\author{
By: Giovanni E. Reyes - Isabella Loaiza Saa
}

\begin{abstract}
Taking into account main features from the immediate Colombian economic and social history, namely from 2000 to 2013, this study analyses relationships among major economic variables, especially economic growth and international trade links. In order to present a more complete picture, this research incorporates the study of annual rate of inflation and rates of unemployment, as well. Colombian economic policy, mostly since the implementation of political measures based on the "Washington Consensus" has reinforced the role of exports as a crucial factor to obtain economic growth. The main argument of this research is that -rather than the quantum of exports- is the price of products in the international area the primary responsible for improving international trade relationships.
\end{abstract}

Key Words: international trade links, Colombian economy, Colombian social and economic conditions.

JEL Classification: E01, E10, F14. 


\section{CRECIMIENTO ECONÓMICO Y COMERCIO INTERNACIONAL}

La finalidad básica de este estudio, es presentar un análisis respecto a la relación entre comercio internacional y crecimiento económico en Colombia, durante el período de 2000 a 2013. Con el fin de caracterizar al crecimiento económico como una variable dependiente, pero no sólo de la influencia del comercio internacional, se incluyen aquí otras dos variables que conforman -conjuntamente con el desempeño económico- el grupo de las variables vitales de la economía: inflación y desempleo.

La influencia del comercio internacional en el crecimiento económico ha sido extensamente estudiada y forma parte del contenido de estudios económicos clásicos en cuanto a: (i) desempeño económico -Smith, Ricardo y Marx; (ii) teoría del valor -Ricardo, Walras y Shumpeter; (iii) relación con sostenibilidad y pleno empleo -Keynes, Friedman y Hayek; (iv) ciclos de causación acumulativa y desarrollo -en general la corriente post-keynesiana, Krugman, Myrdal, Stiglitz; y (v) neoinstitucionalisimo -principalmente North (Smith, 2004; Ricardo, 2007; Krugman 2007)2 .

Considerando que el crecimiento o desempeño económico es resultante de un conjunto complejo de factores interrelacionados, es que se ampliaron, más allá del comercio internacional, las variables exógenas o independientes. En todo caso, en el cálculo del coeficiente o intercepción, en los modelos de regresión múltiple se captura la influencia de los otros factores que como variables exógenas, no se han hecho explícitas en el modelo. Los datos pertenecientes al último semestre de 2013 se basan en proyecciones realizadas con base en datos del Banco de la República y Proexport de Colombia (Banco de la República de Colombia, 2003) ${ }^{3}$.

Las caracterizaciones estadísticas y econométricas que aquí se incluyen tienen una finalidad básicamente descriptiva. No pueden ser totalmente concluyentes, aunque si muestran tendencias, debido al número de grados de libertad con los que se trabajó. Al tomar en cuenta datos semestrales, desde enero de 2000, son 14 años, que equivalen a 28 semestres $^{4}$.

2. Dentro de las obras y los comentarios, ensayos o actualizaciones más representativas: Smith, Adam (2004) Teoría de los Sentimientos Morales. (México, D.F., México: Fondo de Cultura Económica); Smith, Adam \& Hayek, Friedrich (2007) Ética y Economía. (México, D.F., México: Editorial de la Universidad Iberoamericana); Ricardo, David (2007) Ricardo's High Price of Bullions and an Essay on Profits. (Nueva York: Cosimo Publs.); Arestis, Philip (2004) Crítica a la Economía Ortodoxa. (Barcelona, España: Universidad Autónoma de Barcelona); Krugman, Paul (2007) Rethinking International Trade. (Massachusetts: MIT Press); Myrdal, Gunnar (2004) Asian Drama: An Inquiry into the Poverty of Nations. (Nueva Delhi: India: Kalyani Publishers); Stiglitz, Joseph (2008) Los Felices Noventa y la Semilla de la Destrucción. (Bogotá, Colombia: Taurus); Stiglitz, Joseph (2002) El Malestar con la Globalización. (Bogotá, Colombia: Taurus); North, Douglass. (1981). Structure and Change in Economic History. (New York: W. W. Norton \& Co.); y North, Douglass. (2008). Institutions, Institutional Change and Economic Performance. (New York: Cambridge University Press).

3. Véase: Banco de la República de Colombia (2003) Comercio Exterior y Actividad Económica de Colombia. (Bogotá, Colombia: Banco de la República, Serie de Publicaciones, Borradores de Economía No. 163; http://www.banrep.gov.co/es/borrador163); Proexport: (http://www.proexport.com. co/publicaciones).

4. En general se estima que para llegar a validación de datos, procesos de inferencia estadística y de comprobación de hipótesis, el número de grados de libertad debe ser como mínimo igual o superior 


\section{Gráfico 1}

EXPORTACIONES E IMPORTACIONES

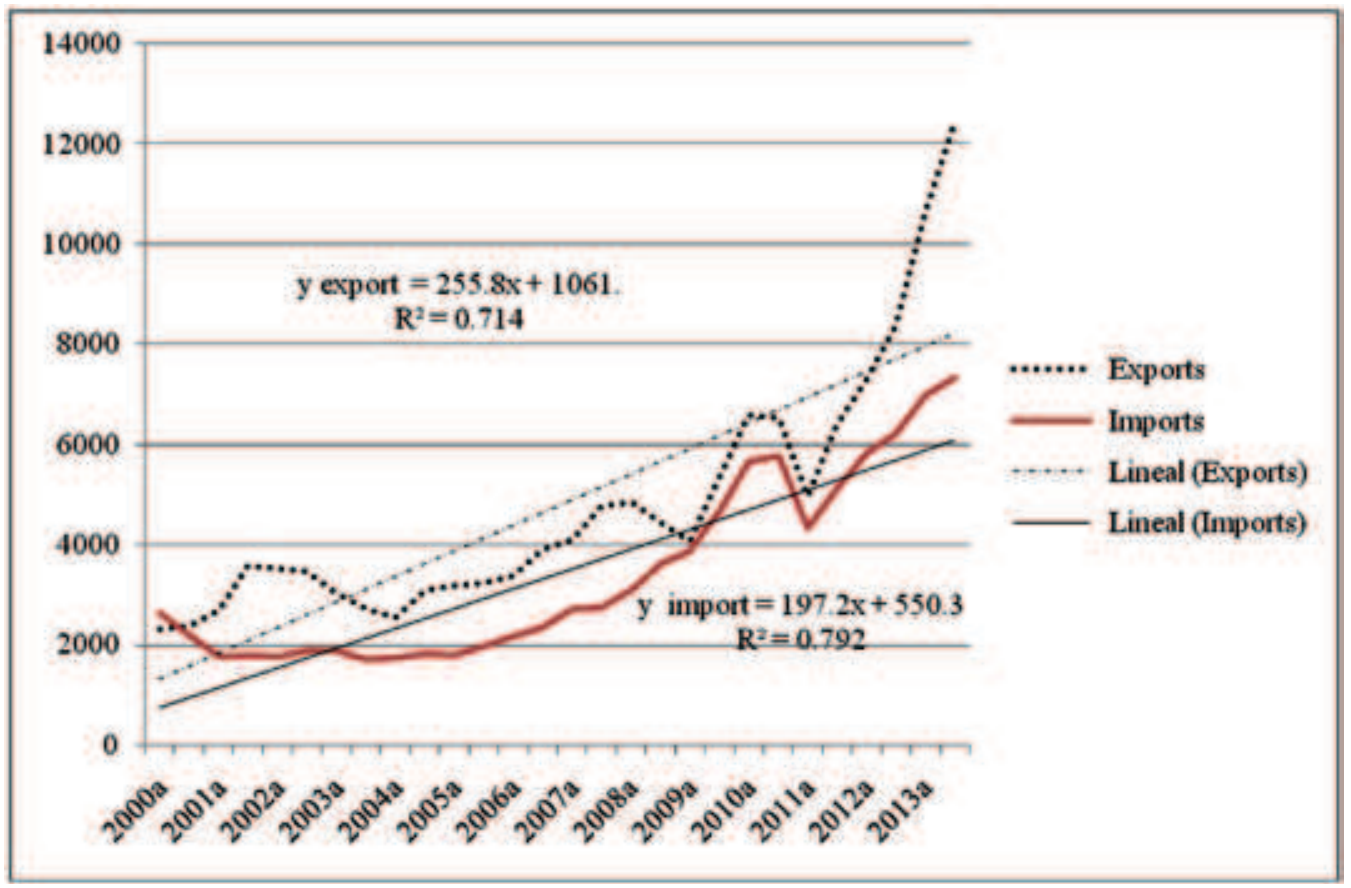

Fuente: Cálculos de este estudio.

En una primera etapa del estudio se caracterizaron gráficamente, las variables a estudiar -crecimiento económico, exportaciones, importaciones, inflación y desempleo-. A continuación se determinaron las principales estadísticas por cada variable.

En una segunda fase se estableció una matriz de correlaciones $\left(n{ }^{*} \mathrm{n}\right)$ a partir de la cual nos aproximamos a las tendencias que se tienen entre el comportamiento de las diferentes variables. Allí se incluyeron en los cálculos los coeficientes de correlación de Pearson que presentaban significancia estadística -ya sea a 1, 5 o 10 por ciento-.

En una tercera etapa se determinaron modelos de regresión múltiple teniendo como variable dependiente o endógena al crecimiento económico. En estos modelos se estableció la influencia conjunta de las variables (coeficiente de Fisher y su significancia estadística), los valores de "t” o "student" para determinar la repercusión

a 10 por cada variable independiente o exógena, a ser incluida en el modelo. Mayor discusión conceptual en Levin, Jack (2008) Introducción a Estadística en Investigación Social. (México, D.F.,

México: Harla); García, Luis (2010) Econometría de Evaluación de Impacto. (Lima, Perú: Pontificia

Universidad Católica del Perú); y Canales, Manuel (2008) Metodologías de la Investigación Social. (Santiago de Chile, Chile: LOM, Ediciones). 
de cada variable exógena en el modelo, el coeficiente de determinación (relacionado con el nivel explicativo del modelo) y una medición de autocorrelación, en el coeficiente de Durbin-Watson.

Finalmente, en una cuarta etapa, se realizó un análisis por agrupaciones (“cluster analysis”). Producto de la aplicación de este procedimiento, se identificó un dendrograma o mapa de densidades en función de las similitudes de las medias del comportamiento, para cada semestre, de los valores presentados por cada una de las cinco variables en estudio (Levin 2008; García 2010) ${ }^{5}$.

En el Gráfico 1, se presenta el comportamiento de las exportaciones e importaciones colombianas en los pasados 14 años. Es posible advertir que desde 2000 hasta 2008 aproximadamente se tuvo un comportamiento paralelo en exportaciones e importaciones. En todo ese período se hace evidente que la balanza comercial del país fue positiva. A partir de 2008 la situación de exportaciones aumenta más que las importaciones. Esto se debe, más que al quantum o cantidad de los productos vendidos en el exterior, a los precios que las exportaciones están teniendo en los mercados foráneos.

Las tasas semestrales de comportamiento respecto a desempleo, inflación y crecimiento económico están contenidas en el Gráfico 2; el desempleo en general ha bajado de manera consistente desde 2002.

A partir de 2008 el crecimiento económico ha superado las tasas de inflación en el país. Una situación contraria se tenía a inicios de la primera década del Siglo XXI. Es notable, por otra parte, cómo en 2005 y 2006, el nivel de crecimiento económico no repercutió tanto como se esperaría, en los niveles de desempleo del país, los cuales continuaron con una tendencia a la baja, pero no de manera dramática.

Los resultados del cálculo de modelos de tendencia lineal, así como coeficientes de determinación -que identifican el ajuste de los datos- se muestran a continuación:

$$
\begin{array}{lll}
\text { Crecimiento económico: } & \mathrm{y}=0.026 \mathrm{x}+3.70 & \mathrm{R}^{2}=0.012 \\
\text { Desempleo: } & \mathrm{y}=-0.313 \mathrm{x}+18.41 & \mathrm{R}^{2}=0.862 \\
\text { Inflación: } & \mathrm{y}=-0.212 \mathrm{x}+8.16 & \mathrm{R}^{2}=0.687
\end{array}
$$

En esos modelos la variable y está referida a los años o tiempo. El modelo que presenta menor volatilidad, es decir más consistencia en el desempeño es el desempleo. En el otro extremo, el crecimiento económico tiene mayor volatilidad. En cuanto a las tendencias, tanto el desempleo como la inflación tienden a la baja; con mayor coeficiente el desempleo que la inflación. No sólo mayor coeficiente sino con menor volatilidad, como fue indicado.

5. Mayores discusiones conceptuales y aplicaciones de análisis por agrupaciones en Romesburg, Charles (2004) Cluster Analysis for Researchers. (North Carolina, United States: Wadsworth Publs); Everitt, Brian, et. al. (2011) Cluster Analysis: Probability and Statistics. (New York: Wiley); Abonyi, Janos (2007) Cluster Analysis for Data Mining and Systemic Identification. (Basel, Germany: Pannonia). 
Gráfico 2

TASAS DE CRECIMIENTO ECONÓMICO, INFLACIÓN Y DESEMPLEO

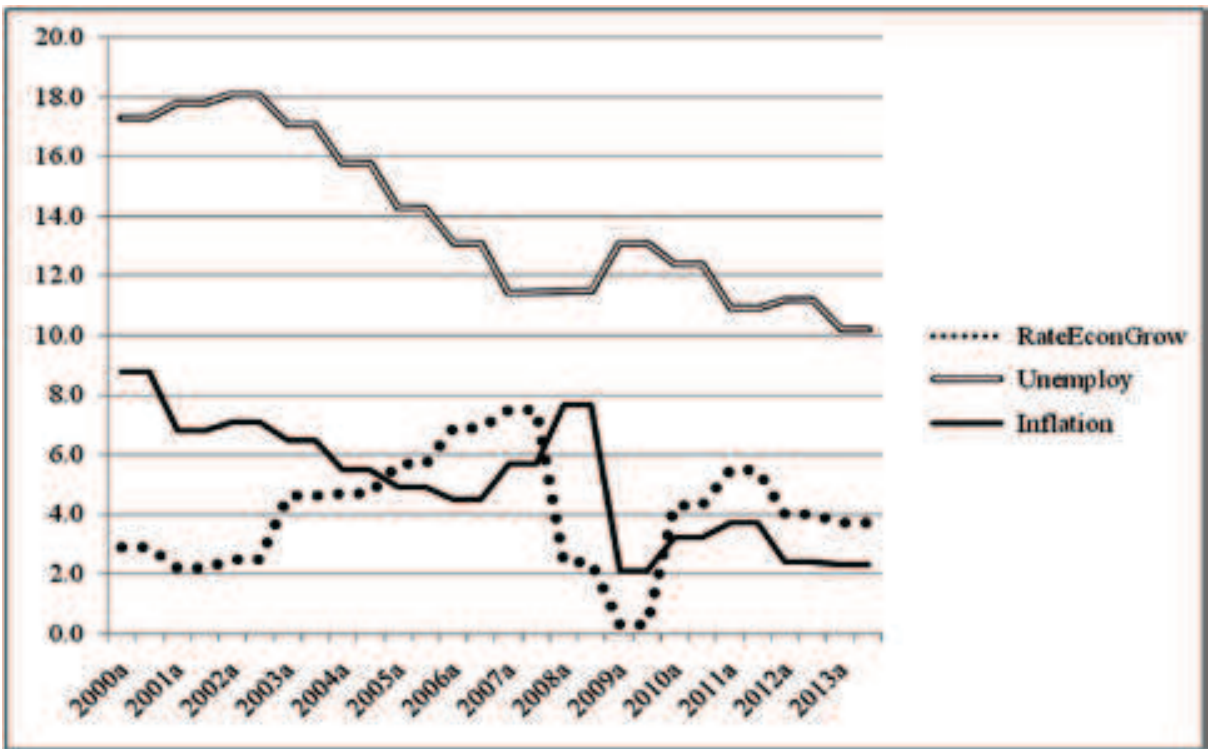

Fuente: Cálculos de este estudio.

Tabla 1

COLOMBIA 2000-2013: CRECIMIENTO ECONÓMICO Y VARIABLES MACROECONÓMICAS ESTADÍSTICOS DESCRIPTIVOS

\begin{tabular}{lccccc}
\hline \multicolumn{1}{c}{ Indicador } & $\begin{array}{c}\text { Crecimiento } \\
\text { Económico }\end{array}$ & Exportación & Importación & Inflación & Desempleo \\
\hline Media aritmética & 4.08 & 4770 & 3409 & 5.08 & 13.87 \\
Mediana & 4.15 & 3975 & 2678 & 5.20 & 13.10 \\
Máximo & 7.50 & 12439 & 7340 & 8.80 & 18.10 \\
Mínimo & 0.30 & 2298 & 1706 & 2.10 & 10.20 \\
Rango & 7.20 & 10141 & 5634 & 6.70 & 7.90 \\
Desv. Estándar $/{ }^{1}$ & 1.91 & 2489 & 1822 & 2.10 & 2.77 \\
Coef. deVariac. ${ }^{2}$ & 0.46 & 0.52 & 0.53 & 0.41 & 0.19 \\
\hline
\end{tabular}

Notas: 1/ desviación estándar de la muestra, esto es con el coeficiente de corrección de Pearson en el denominador $(n-1)$;

2/ Coeficiente de variación de conformidad con: \{[(Desv. Est. / Med. Aritmética) $] * 100\}$.

Fuente: Cálculos de este estudio. 
En la Tabla 1, con la caracterización general a partir de los indicadores estadísticos, se confirman varias tendencias. Las exportaciones tienden a ser mayores que las importaciones, de conformidad con las cifras de media aritmética, mediana así como los valores máximos. El valor mayor de la desviación estándar de las exportaciones indica que esta variable tiene mayor volatilidad que las importaciones, aún cuando los valores de los coeficientes de variación tienden a mostrar diferencias matemáticas, más no estadísticas.

En lo referente al empleo la situación ha sido preocupante en la economía colombiana. Colombia es, junto con Uruguay, Panamá y Perú, de los países que menos han sido impactados por la última crisis económica financiera internacional. La crisis que tuvo su punto de inflexión el 13 de septiembre de 2008 con la quiebra del banco de inversión Lehman Brothers.

Es de considerar que para que el crecimiento económico tenga una repercusión positiva en el potencial de desarrollo, debe promover esencialmente el empleo. En la medida que se incrementan puestos de trabajo en la economía real -la que se relaciona con la producción de bienes, servicios y empleo- se ven aumentadas las oportunidades para la población.

Si a este mayor número de mejoras en las posibilidades laborales se agregan también ampliaciones en las capacidades de las personas -vía la educación y la capacitación- se fortalece la potencialidad real de consolidar sostenidamente mayores niveles de desarrollo económico y social.

Mediante la matriz de correlaciones de la Tabla 2, podemos aproximarnos a la determinación de las tendencias que hay en el desempeño de las variables y saber si las mismas tienen o no significancia estadística. Por ejemplo, las exportaciones y las importaciones tienen una correlación de 92.9 por ciento con alta significancia estadística. Esto mismo puede decirse, en un sentido inverso, entre exportaciones y desempleo -también con un atributo de significado estadístico.

Es decir, que en la medida que las exportaciones se mantienen con ritmos dinámicos, se esperaría la creación de mayores puestos de trabajo. También se puede evidenciar una correlación inversa entre exportaciones e inflación. A medida que los mercados internacionales demuestran mayor capacidad de absorción de los productos colombianos, la inflación tendería a estar bajo mayor control.

De manera similar, un mayor dinamismo de las importaciones tiende a generar menores niveles de desempleo y tienden a decrecer los niveles de aumentos generalizados de los precios de bienes y servicios, esto es, índices inflacionarios.

Las tasas anuales de crecimiento no muestran tener correlaciones significativas con ninguna de las variables bajo estudio. Es decir que aunque se pueden detectar tendencia matemáticas, las mismas no tienen significancia estadística al trabajar con niveles de confianza de 1 o bien 5 por ciento.

De manera complementaria, a mayor nivel de inflación en ciertos períodos específicos del total de 14 años del estudio, se pudo apreciar cierta ampliación del desempleo, aunque en general esta tendencia mostró una tendencia a la baja. Con ello, se puede puntualizar que Colombia no estaría inmune a mostrar escenarios de estanflación en Colombia, una combinación de estancamiento económico identificado con cifras relativamente altas de desempleo, a la vez que se tienen índices significativos de inflación. 
Giovanni E. Reyes - Isabella Loaiza Saa

Tabla 2

MATRIZ DE CORRELACIONES

\begin{tabular}{|c|c|c|c|c|c|c|}
\hline & & Exportación & Importación & TasaDeCreEc & Desempleo & Inflación \\
\hline \multirow{3}{*}{ Exportación } & Correlación de Pearson & 1 & $929 * *$ &,- 030 &,$- 720^{* *}$ &,$- 694^{* *}$ \\
\hline & Sig. (bilateral) & &, 000 & 881 & ,000 &, 000 \\
\hline & $\mathrm{N}$ & 28 & 28 & 28 & 28 & 28 \\
\hline \multirow{4}{*}{ Importación } & Correlación de Pearson &, $929 * *$ & 1 &,- 119 &,$- 755^{* *}$ &,$- 743^{* *}$ \\
\hline & Sig. (bilateral) & ,000 & & ,546 & ,000 &, 000 \\
\hline & $\mathrm{N}$ & 28 & 28 & 28 & 28 & 28 \\
\hline & Correlación de Pearson &,- 030 &,- 119 & 1 &,- 295 &,- 071 \\
\hline \multirow[t]{2}{*}{ TasaDeCreEc } & Sig. (bilateral) & ,881 & ,546 & & 127 & ,720 \\
\hline & $\mathrm{N}$ & 28 & 28 & 28 & 28 & 28 \\
\hline \multirow{3}{*}{ Desempleo } & Correlación de Pearson &,$- 720^{* *}$ &,$- 755^{* *}$ &,- 295 & 1 &, $667^{* *}$ \\
\hline & Sig. (bilateral) & ,000 &, 000 & 127 & &, 000 \\
\hline & $\mathrm{N}$ & 28 & 28 & 28 & 28 & 28 \\
\hline \multirow{3}{*}{ Inflación } & Correlación de Pearson &,$- 694^{* *}$ &,$- 743^{* *}$ &,- 071 &, $667^{* *}$ & 1 \\
\hline & Sig. (bilateral) & ,000 & ,000 & 720 & ,000 & \\
\hline & $\mathrm{N}$ & 28 & 28 & 28 & 28 & 28 \\
\hline
\end{tabular}

**. La correlación es significativa al nivel 0,01 (bilateral).

Fuente: Cálculos de este estudio.

Modelo 1

VAR. ENDÓGENA: CRECIMIENTO ECONÓMICO VAR. EXÓGENAS: EXPORTACIÓN, IMPORTACIÓN, INFLACIÓN Y DESEMPLEO

\begin{tabular}{|c|c|c|c|c|c|c|}
\hline Tasa de Crec. Ec. & $=$ & 15.78 & $+367(10)^{-6}$ Expor. & -0.0013 Imp. & -0.143 Infl. & -0.579 Des. \\
\hline Valores t & & $(4.78)$ & $(1.08)$ & $(-2.70)$ & $(-0.62)$ & $(-3.29)$ \\
\hline Signific. Estad. & & $* * *$ & & $* * *$ & & $* * *$ \\
\hline $\mathrm{R}^{2}$ & $=$ & 0.41 & & & & \\
\hline $\mathrm{F}$ & $=$ & $3.83 * * *$ & & & & \\
\hline Durbin-Watson & $=$ & 0.88 & & & & \\
\hline $\mathrm{N}$ & $=$ & 28 & & & & \\
\hline
\end{tabular}

En el Modelo 1, se incorporan las dos variables de comercio exterior como componentes independientes de la cuantificación. Como se puede apreciar, el nivel explicativo total del modelo es de 41 por ciento. El impacto conjunto de las variables independientes o exógenas da un coeficiente $\mathrm{F}$ que es significativo estadísticamente con un nivel de error de 1 por ciento. 
El coeficiente de Durbin-Watson al tener valores menores del coeficiente 1, da muestras de tener niveles notables de autocorrelación. Esto se debería a que las exportaciones e importaciones presentan este rasgo. Es por ello que se calcularon los modelos 2 y 3 . Como se puede apreciar, en estos últimos, en cada uno de ellos, se incorpora sólo las exportaciones y sólo las importaciones como variables independientes.

Con este procedimiento se evitaría el "ruido" estadístico de la autocorrelación que se ha podido evidenciar con el coeficiente Durbin-Watson ${ }^{6}$.

De conformidad con los resultados del Modelo 2, se mantiene aún la situación de autocorrelación del coeficiente Durbin-Watson, no osbtante este rasgo y la dificultad de los grados de libertad (g.l.) que le imprime a esta cuantificación un carácter descriptivo y de tendencias, las exportaciones sí tienen un efecto, con un 10 por ciento de nivel de confianza, en cuanto a su repercusión favorable -relación directa- con el crecimiento económico. El nivel de desempleo muestra una relación, tal y como se esperaba, inversa y significativa al 5 por ciento, en referencia al crecimiento económico.

Modelo 2

VAR. ENDÓGENA: CRECIMIENTO ECONÓMICO VAR. EXÓGENAS: EXPORTACIÓN, INFLACIÓN Y DESEMPLEO

\begin{tabular}{llllll}
\hline Tasa de Crec. Ec. & $=$ & 12.12 & $+374(10)^{-6}$ Expor. & +0.035 Infl. & -0.464 Des. \\
Valores t & & $(3.58)$ & $(-1.68)$ & $(0.14)$ & $(-2.41)$ \\
Signific. Estad. & & $* * *$ & $*$ & & $*$ \\
$\mathrm{R}^{2}$ & $=$ & & & \\
$\mathrm{F}$ & $=$ & & & \\
Durbin-Watson & $=$ & & & \\
$\mathrm{N}$ & $=$ & & & & \\
\hline
\end{tabular}

Notas: $1 /$ significancias estadísticas: ${ }^{*}, 0.10 ;{ }^{* *} 0.05 ;{ }^{* * *} 0.01$

Fuente: cálculos de este estudio.

6. El coeficiente Durbin-Watson, con su determinación de la posible autocorrelación de variables, fue basado en investigaciones matemáticas de John von Neumann -pionero de la "teoría de juegos". Durbin-Watson se basa en el siguiente modelo:

$$
d=\frac{\sum_{t=2}^{T}\left(e_{t}-e_{t-1}\right)^{2}}{\sum_{t=1}^{T} e_{t}^{2}}
$$

El cálculo del coeficiente se basa en valores de secuencia, donde se toman en cuenta la evidencia del posible efecto de rezago o inercia, referente a los residuales de un modelo de mínimos cuadrados. Si los valores bajan de 1 es muy probable haber detectado un fenómeno de autocorrelación. El valor de 2 indicaría la ausencia de autocorrelación. Mayores discusiones en: Anderson, David; et. al. (2003) Estadística para Administración y Economía. (México, D.F., México: Thompson Editores) en especial, pp. 87-94. 
En el Modelo 3, son las importaciones, como único componente del comercio internacional, la variable que se toma en consideración como componente exógeno o independiente. El coeficiente Durbin-Watson mejora un tanto, pero no supera el coeficiente 1, con lo que se reafirma el rasgo descriptivo del modelo, de identificación de tendencias.

Modelo 3

VAR. ENDÓGENA: CRECIMIENTO ECONÓMICO VAR. EXÓGENAS: IMPORTACIÓN, INFLACIÓN Y DESEMPLEO

\begin{tabular}{llllll}
\hline Tasa de Crec. Ec. & $=$ & 16.195 & $-928(10)^{-6}$ Imp. & -0.139 Infl. & -0.593 Desemp. \\
& & $(4.92)$ & $(-3.10)$ & $(-0.61)$. & $(-3.37)$ \\
Valores t & & $* * * / 1$ & $* * *$ & & \\
Signific. Estad. & & 0.36 & & & \\
$\mathrm{R}^{2}$ & $=$ & & & \\
$\mathrm{F}$ & & & & \\
Durbin-Watson & $=$ & & & \\
$\mathrm{N}$ & $=$ & & & & \\
\end{tabular}

Notas: $\quad 1 /$ significancias estadísticas: *, 0.10; **0.05; **0.01

Fuente: Cálculos de este estudio.

En el Modelo 3 en contraste con el Modelo 2, el coeficiente de Fisher demuestra tener significancia estadística al 1 por ciento de error. El poder explicativo del modelo en su conjunto es de 36 por ciento según se puede inferir del valor del coeficiente de determinación.

Las importaciones demuestran tener una relación inversa con el crecimiento económico, con nivel o intervalo de confianza de 1 por ciento. Una situación similar, también en el sentido inverso, se ha podido detectar entre la variable dependiente y el nivel de desempleo.

El Gráfico 3 contiene el resultado de un análisis por agrupaciones o "clusters". Partiendo del extremo izquierdo, cada uno de los semestres estudiados forman por si mismos un grupo. En la medida que nos movemos, dentro del dendrómetro hacia la derecha, se van integrando los grupos, hasta la situación extrema en la cual todos los sub-grupos forman un conjunto total.

Uno de los rasgos más llamativos del análisis por agrupaciones es que los últimos cuatro años, a partir del segundo semestre de 2008 o más propiamente comenzando con el primer semestre de 2009, se tienen períodos distintos a los semestres anteriores de la serie que comenzó en enero de 2000. Esta situación se ve muy influenciada por el comportamiento de las exportaciones e importaciones.

Como fue posible advertir desde la caracterización general de las gráficas y los indicadores estadísticos básicos, a partir de 2008 la situación de exportaciones e importaciones ha mostrado un gran dinamismo. Como fue referido con anterioridad, uno de los factores más importantes en esto ha sido el alto precio de los productos colombianos en el exterior -esto es la factura de productos- en especial los valores de minería que conforman, para 2013 un 75 por ciento del total de exportaciones del país. 


\section{Gráfico 3}

Dendrograma que utiliza una vinculación media (entre grupos) Combinación de conglomerados de distancia re-escalados

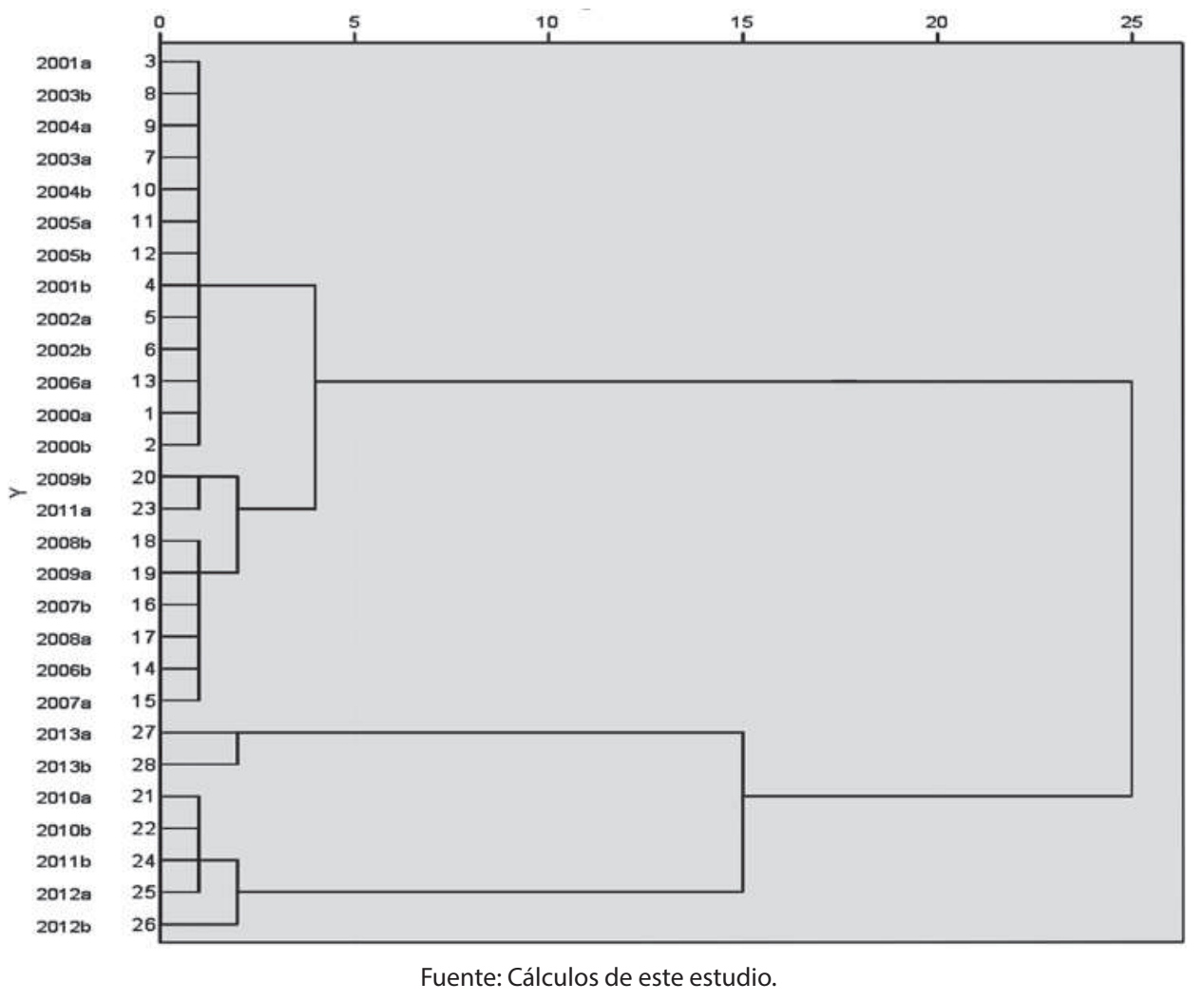

El resultado positivo en la balanza comercial ha sido posible no obstante que la tasa de cambio del peso colombiano, durante los pasados ocho años, haya estado en rangos de notable apreciación en relación con el dólar estadounidense. Como se sabe, en la medida que una moneda muestra altos niveles de cotización o "fortaleza" en relación con una moneda referencial, se tienden a desestimular las exportaciones, en tanto las importaciones, al ser más baratas, compiten por la demanda agregada del mercado doméstico en el país ${ }^{7}$.

7. Véase al respecto, una muy reciente síntesis teórica en esta relación entre política cambiaria, tasas de cambio monetario, balanza comercial y cuenta corriente de la balanza de pagos, en Nicita, Alessandro (2013) Exchange Rates, International Trade and Trade Policies. (Geneva, Switzerland: United Nations Conference on Trade and Development, UNCTAD); otras fuentes respecto a fundamentación teórica y discussion de casos en: Rajan, Raghuram and Subramanian, Arvind, (2011). Aid, Dutch Disease, and Manufacturing Growth. Journal of Development Economics. 94(1): 106-118; Rodrik, Dani, (2008). The Real Exchange Rate and Economic Growth. (New York: Brookings Papers on Economic Activity) y Kee, Hiau Looi, Alessandro Nicita and Marcelo Olarreaga, (2008). Import demand elasticities and trade distortions. The Review of Economics and Statistics. 90(4): 666-682. 
Finalmente, como pruebas complementarias, se presentan en el anexo, el comportamiento de valores marginales en función de gráfica de residuos, estimados observados y datos adjuntados. También se incluye un gráfico sobre el comportamiento de la normalidad de parámetros, teniendo como referencia la variable dependiente de tasas de variación semestral del producto interno bruto de Colombia.

\section{CONCLUSIONES Y CONSIDERACIONES FINALES}

El análisis de la relación entre crecimiento económico de Colombia y comercio internacional se realizó para el período 2000 - 2013, incluyendo la caracterización de otras variables vitales en la economía de un país, además de la tasa de variación del producto interno bruto, esto es: inflación y desempleo.

A partir de 2008 y como un factor que ha permitido evitar hasta ahora los efectos de la última crisis financiera internacional, ha tendido a existir un notable incremento en el dinamismo de exportaciones e importaciones. Eso ha sido posible no obstante la alta cotización del peso colombiano respecto al dólar estadounidense.

En general la balanza comercial, durante los 14 años del estudio, ha tendido a mantenerse en rangos positivos. El desempleo ha tendido consistentemente a la baja durante toda la serie analizada, no obstante que -en comparación con otros países latinoamericanos- sus valores aún son relativamente elevados.

El factor principal en este dinamismo han sido los relativamente altos precios de las exportaciones del país en los mercados internacionales. De allí la preocupación de que una baja en esos precios en los mercados mundiales, puede implicar ciertos niveles de desaceleración económica.

Tal y como es posible esperar de una economía que se desempeña en condiciones generalizadas, el crecimiento económico ha implicado una baja en los niveles de desempleo, pero ese descenso, no obstante no ha sido significativo. Esto tiene el inconveniente de que va asegurando que una de las principales correas transmisoras entre crecimiento económico y desarrollo, no está desempeñándose en los niveles que son deseables. El crecimiento económico observado en el país no está abriendo, tanto como sería predecible, los aumentos de oportunidades para la población vía el incremento de puestos de trabajo en la economía real. 
BIBLIOGRAFÍA

ABONYI, Janos (2007). Cluster Analysis for Data Mining and Systemic Identification. Basel, Germany: Pannonia.

ANDERSON, David; et. al. (2003). Estadística para Administración y Economía. México, D.F., México: Thompson Editores.

ARESTIS, Philip (2004). Crítica a la Economía Ortodoxa. Barcelona, España: Universidad Autónoma de Barcelona.

BANCO DE LA REPÚBLICA DE COLOMBIA (2003). Comercio Exterior y Actividad Económica de Colombia. Bogotá, Colombia: Banco de la República, Serie de Publicaciones, Borradores de Economía No. 163; http:// www.banrep.gov.co/es/borrador163.

CANALES, Manuel (2008). Metodologías de la Investigación Social. Santiago de Chile, Chile: LOM, Ediciones. EVERITT, Brian, et. al. (2011). Cluster Analysis: Probability and Statistics. New York: Wiley.

GARCíA, Luis (2010). Econometría de Evaluación de Impacto. Lima, Perú: Pontificia Universidad Católica del Perú.

KEE, Hiau Looi; NICITA, Alessandro and OLARREAGA, Marcelo (2008). Import demand elasticities and trade distortions. The Review of Economics and Statistics. 90(4): 666-682.

KRUGMAN, Paul (2007). Rethinking International Trade. Massachusetts: MIT Press.

LEVIN, Jack (2008). Introducción a Estadística en Investigación Social. México, D.F., México: Harla.

MYRDAL, Gunnar (2004). Asian Drama: An Inquiry into the Poverty of Nations. Nueva Delhi: India: Kalyani Publishers.

NICITA, Alessandro (2013). Exchange Rates, International Trade and Trade Policies. Geneva, Switzerland: United Nations Conference on Trade and Development, UNCTAD.

NORTH, Douglass (1981). Structure and Change in Economic History. New York: W. W. Norton \& Co.

NORTH, Douglass (2008). Institutions, Institutional Change and Economic Performance. New York: Cambridge University Press.

RAJAN, Raghuram \& SUBRAMANIAN, Arvind, (2011). Aid, Dutch Disease, and Manufacturing Growth. Journal of Development Economics. 94(1): 106-118.

RICARDO, David (2007). Ricardo's High Price of Bullions and an Essay on Profits. Nueva York: Cosimo Publs. RODRIK, Dani (2008). The Real Exchange Rate and Economic Growth. New York: Brookings Papers on Economic Activity.

ROMESBURG, Charles (2004). Cluster Analysis for Researchers. North Carolina, United States: Wadsworth Publs.

SMITH, Adam \& HAYEK, Friedrich (2007). Ética y Economía. México, D.F., México: Editorial de la Universidad Iberoamericana.

SMITH, Adam (2004). Teoría de los Sentimientos Morales. México, D.F., México: Fondo de Cultura Económica. STIGLITZ, Joseph (2002). El Malestar con la Globalización. Bogotá, Colombia: Taurus.

STIGLITZ, Joseph (2008). Los Felices Noventa y la Semilla de la Destrucción. Bogotá, Colombia: Taurus. 
ANEXO

Gráfico 1

COMPORTAMIENTOS MARGINALES: RESIDUOS, REALES Y AJUSTADOS

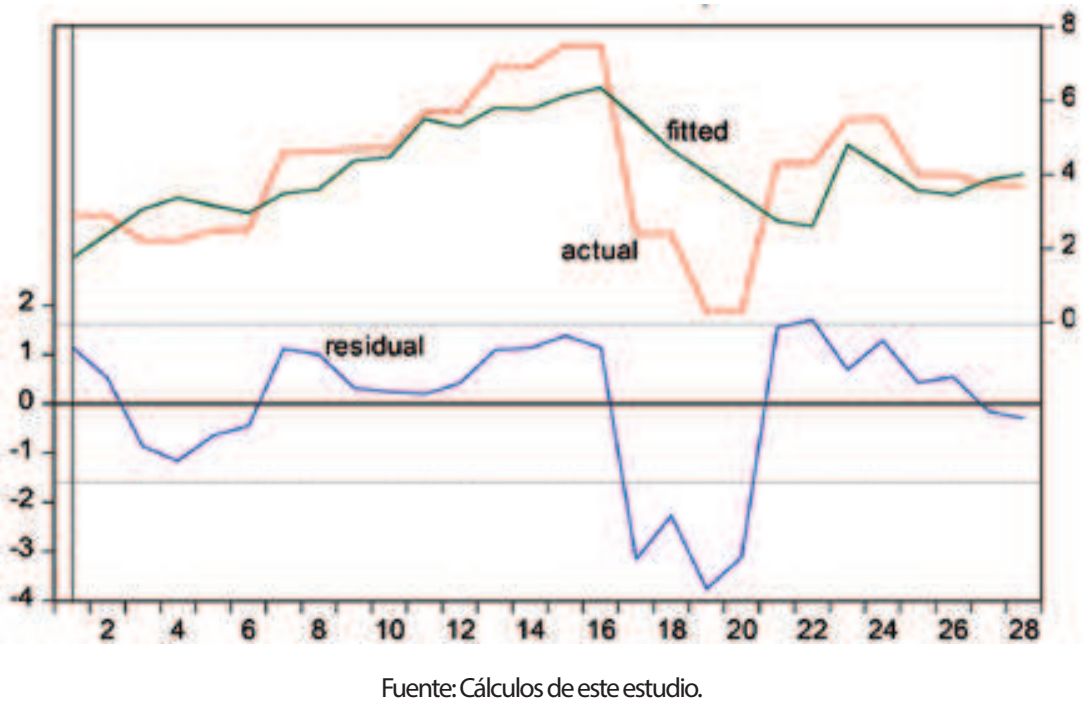

Gráfico 2

HISTOGRAMA

Variable dependiente: TasaDeCreEc

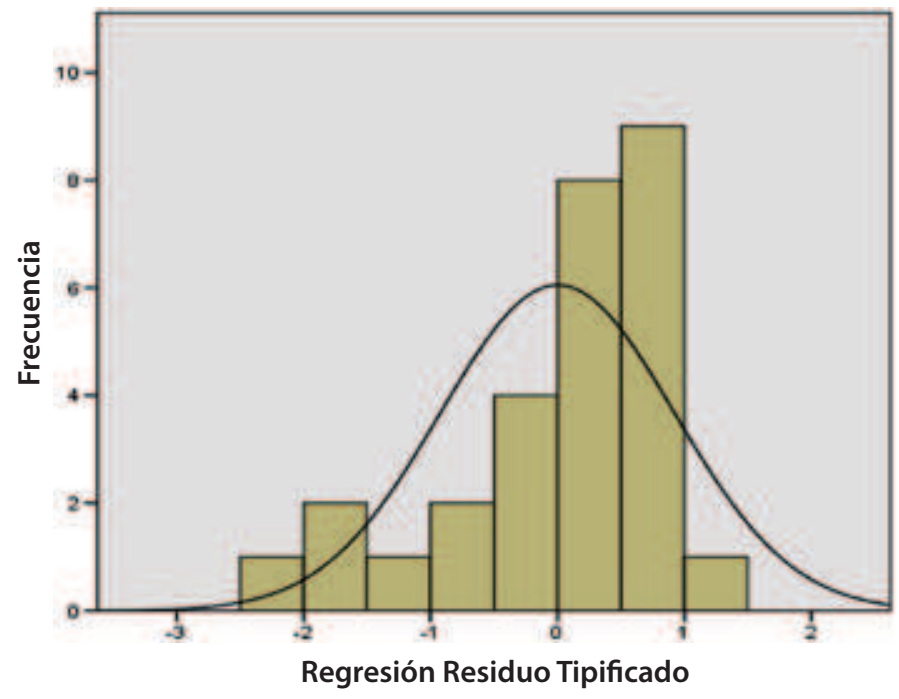

Media $=2,32 \mathrm{E}-16$

Desviación típica $=0,923$

$\mathrm{N}=\mathbf{2 8}$

Fuente: Cálculos de este estudio. 